\title{
Developmental Expression of REGA-1, a Regionally Expressed Glial Antigen in the Central Nervous System of Grasshopper Embryos
}

\author{
Ellen M. Carpenter and Michael J. Bastiani \\ Department of Biology, University of Utah, Salt Lake City, Utah 84112
}

Glial cells are a large component of the developing nervous system, appearing before the onset of axon outgrowth in a variety of developing systems. Their time of appearance and their location in conjunction with developing axon pathways may allow them to define the position of axon pathways. Specific glial cells may be utilized as guideposts by growing axons, allowing them to recognize the appropriate pathway, or conversely, glial cells may inhibit axons from growing along an inappropriate pathway. The 7F7 monoclonal antibody labels a subset of glial cells in grasshopper embryos that may play a role in defining the location of selected axonal pathways. This antibody recognizes the REGA-1 molecule, a cell-surface antigen with a molecular weight of 60 $\mathrm{kDa}$, which is regionally expressed on developing glial cells. REGA-1 is expressed around the edges of clusters of glial cells and on lamellae extending from glial cells to line the edges of some axonal pathways. REGA- 1 expression is first seen in the neuroblast sheet, surrounding neuroblast 4-1. Slightly later in development, 2 glial cells extend processes that express REGA-1 and demarcate the caudal edge of the anterior commissure. As the animal matures, cell processes expressing REGA-1 line the edges of the longitudinal connective, then expand to surround the central neuropil of the segmental ganglia. REGA-1 expression is also seen in conjunction with axons leaving the segmental ganglia via the segmental nerves and the intersegmental connectives. REGA-1 expression is limited to a subset of glial cells; some known glial cells such as the segment boundary cell do not express REGA-1. Glial cell processes expressing REGA-1 are seen only in association with axons, which suggests that these processes may act as borders or guard rails confining axons to the appropriate regions of the developing CNS. Axons navigating a path through the CNS may be prohibited from growing into inappropriate regions based on their inability to cross the boundaries established by glial cells expressing REGA-1.

During the development of the CNS growing axons encounter a wide variety of cellular and noncellular substrates that may provide pathway cues for developing neurons. Glial cells form

\footnotetext{
Received July 9, 1990; accepted Sept. 14, 1990.

We thank Mark R. Meyer for providing the $5 \mathrm{~B} 12$ monoclonal antibody and Paul Z. Myers, Elaine C. Seaver, Rolf O. Karlstrom, and Mary C. Beckerle for critical reading of the manuscript. This research was supported by NIH Grant NS25387 and by a McKnight Scholars Award to M.J.B. E.M.C. was supported by NIH Postdoctoral Fellowship NS08404.

Correspondence should be addressed to Ellen M. Carpenter, Ph.D., Department of Biology, 201 South Biology, University of Utah, Salt Lake City, UT 84112. Copyright (C) 1991 Society for Neuroscience $0270-6474 / 91 / 010277-10 \$ 03.00 / 0$
}

a large component of the developing CNS, and recent evidence has implicated them in defining the location of developing neural pathways in vertebrates (Silver et al., 1982; Poston et al., 1988) and invertebrates (Bastiani and Goodman, 1986; Jacobs and Goodman, 1989). In vertebrates, glial cells may provide permissive substrates for axonal growth, allowing the formation of such pathways as the corpus callosum (Silver et al., 1982), or they may form boundaries inhibiting axons from entering inappropriate regions (Silver et al., 1987; Poston et al., 1988). Glial cells may provide permissive substrates for axon growth by expressing surface molecules that allow axons to recognize or adhere to them (Silver and Rutishauser, 1984; Johnson et al., 1988). Conversely, glial cells may act to inhibit axon growth into inappropriate regions of the CNS by the expression of inhibitory molecules (Caroni and Schwab, 1988).

Invertebrate glial cells have also been shown to play a role in the formation of axon pathways. Recent observations have suggested that there exists a glial scaffold that marks the presumptive location of axonal pathways before the onset of axon growth in insects (Bastiani and Goodman, 1986; Jacobs and Goodman, 1989). In Drosophila, this scaffold is composed of pairs of elongate glial cells positioned in the regions where the anterior and posterior commissures and the longitudinal connectives will form in the segmental ganglia (Jacobs and Goodman, 1989). Another glial cell, the segment boundary cell, has been observed in association with the developing intersegmental nerve in Drosophila and in grasshoppers (Goodman et al., 1984; Bastiani and Goodman, 1986). During development, axons that pioneer the connectives, commissures, and the intersegmental nerve make extensive contact with these early glial cells. Additionally, experimental manipulations in the grasshopper embryo suggest that the segment boundary cell is required for the correct formation of the intersegmental nerve, because it provides guidance cues necessary for the anterior corner cell ( $\mathrm{aCC}$ ) neuron to grow into the intersegmental nerve (Bastiani and Goodman, 1986). These cues are likely to be manifested by the expression of molecules on the surface of the segment boundary cell that can be recognized by the aCC neuron, allowing it to make an appropriate pathway choice. These observations illustrate that glial cells are positioned appropriately to act in defining the location of axonal pathways in invertebrates, mirroring the observations made in vertebrate systems.

The developmental origins of insect glial cells are largely unknown. It has been suggested that glial cells arise from dedicated glial prccursor cells, elements of the neuroblast sheet whose progeny populate the segmental ganglia as glial cells. One such precursor, whose progeny appear to give rise to some neuropilar glial cells, is located at the lateral edge of the neuroblast sheet (Doe and Goodman, 1985). Alternatively, neuroblasts themselves may be capable of giving rise to glial cells. Such dual 
offspring stemming from a single progenitor have been seen in the development of the vertebrate CNS (Wetts et al., 1989); invertebrate glia may arise in the same manner. Glial cells may also arise from neuroepithelial cells that do not differentiate into neuroblasts. Each neuroblast within the developing neuroepithelium is surrounded by non-neuronal sheath and cap cells (Doe and Goodman, 1985; Doe et al., 1985), which may later develop into glial cells. Additional non-neuronal cells may arise along the midline of the neuroepithelium. Unpaired midline precursor cells, the midline ectodermal cells, give rise to midline precursor (MP) neurons (Bate, 1976; Doe and Goodman, 1985); additionally, they give rise to midline glial cells (Campos-Ortega and Hartenstein, 1985; Jacobs and Goodman, 1989). It is also possible that different morphological types of glial cells may stem from different progenitor populations (Shepherd and Bate, 1990).

Considerably more is known about the location and distribution of glial cells in adult insect ganglia. Glial cells compose a large part of the adult insect CNS and have been classified into various subtypes based on morphological and positional criteria (Wigglesworth, 1959; Lane, 1974; Radojcic and Pentreath, 1979; Hoyle, 1986). Subtypes of glial cells have been recognized that wrap and isolate neuronal somata, that separate the central neuropil from the surrounding neuronal cell bodies, and that are associated with axons in the neuropil, the longitudinal connectives, and the peripheral nerves (Hoyle, 1986).

The early appearance of invertebrate glial cells and their close association with developing axon pathways suggest that glial cells may play a role in specifying the location of these axonal pathways, and that axons must be able to recognize and respond appropriately to glial cells in order to grow along the correct pathway. This suggests that there are likely to be differences among glial cells associated with different pathways. These differences are likely to be manifested by the differential expression of cell-surface molecules that can interact with axons to provide the necessary guidance cues. We have begun to identify and characterize specific non-neuronal cells associated with developing CNS pathways in grasshopper embryos. We have isolated a monoclonal antibody that recognizes the regionally expressed glial antigen-1 molecule (REGA-1) expressed on a subset of glial cells associated with developing axonal pathways. REGA-1 expression on these cells begins early in development in specific regions of the CNS where a nerve pathway will eventually form, preceding the appearance of axons in this pathway. At later stages of development, REGA-1 is expressed in association with other pathways, then expands to line the developing neuropil in the region where neuropilar glial cells are located in adult segmental ganglia. The expression of this antigen on specific glial cells suggests that these cells and, consequently, the expressed antigen may play a role in defining the location of early nerve pathways.

\section{Materials and Methods}

Generation of the $7 F 7$ monoclonal antibody. Ventral nerve cords were dissected from $3055 \%$ grasshopper embryos in to cold Ringer's solution. Nerve cords were homogenized in phosphate-buffered saline (PBS) containing $1 \mathrm{mM}$ phenylmethylsulfonyl fuoride (PMSF) with the addition of protease inhibitors $[\mathrm{P} 1 ; 1 \mu \mathrm{g} / \mathrm{ml}$ each of antipain, chymostatin, leupeptin, pepstatin, $N$-p-tosyl-L-lysine chloromethyl ketone (TLCK), and $N$-tosyl-L-phenylalanine chloromethyl ketone (TPCK)] and centrifuged at $1000 \times g\left(4^{\circ} \mathrm{C}\right)$ for 10 min to separate out nuclei and cellular organelles. The supernatant was centrifuged at $200,000 \times g\left(4^{\circ} \mathrm{C}\right)$ for $30 \mathrm{~min}$, and the resulting membrane pellet was resuspended in $100 \mu \mathrm{l}$ PBS, emulsified with an equal volume of Freund's adjuvant, and injected intraperitoneally into a female Balb/C mouse. The first injection contained Freund's complete adjuvant; subsequent injections were made using Freund's incomplete adjuvant with the exception of the final injection $3 \mathrm{~d}$ prior to fusion, which was without adjuvant. The mouse received 4 injections spaced 2 weeks apart. Spleen cells from the injected mouse were fused with NS-1 myeloma cells using the procedure of Kohler and Milstein (1975). Hybridoma colonies were grown in tissue culture medium consisting of 70\% RPMI 1640 (Cellgro), 20\% bovine calf serum (Hyclone), and 10\% NCTC-135 medium with L-glutamine (Gibco), with the addition of $0.1 \mathrm{~mm}$ nonessential amino acid stock solution (Gibco), $0.2 \mathrm{IU} / \mathrm{ml}$ bovine insulin (Sigma), $1 \mathrm{~mm}$ oxaloacetate (Sigma), and $1 \times$ hypoxanthine-thymidine (HT) solution (Gibco). Undiluted supernatants from the hybridoma colonies were screened on $40 \%$ whole-mount grasshopper embryos using the immunohistochemistry protocol outlined below. The colony secreting the 7F7 monoclonal antibody was selected for further study based on its selective labeling of a very small portion of the CNS in a $40 \%$ grasshopper embryo.

Hybridoma cells that produced the 7F7 antibody were used for the production of ascites fluid. Adult male Balb/C mice were primed with an intraperitoneal injection of Pristane (2,6,10,14-tetramethyl pentadecane; Sigma) 1 week prior to injection of hybridoma cells. Approximately $3 \times 10^{5}$ hybridoma cells were injected intraperitoneally into each primed mouse. Ascites fluid was harvested $10 \mathrm{~d}$ later by tapping the enlarged abdomen of the mouse with a sterile 20 -ga syringe needle. Ascites fluid containing the 7F7 antibody was used for all subsequent experiments.

Immunohistochemistry. For immunohistochemistry of sections through adult ganglia, segmental ganglia were dissected from adult grasshoppers and fixed in $2 \%$ paraformaldehyde in Millonig's buffer. Ganglia were fixed for $4 \mathrm{hr}$, washed in PBS, and immersed overnight in $20 \%$ sucrose. The following day, the ganglia were embedded in liquid gel albumin, hardened, and trimmed to remove excess gelatin. Matrix blocks containing the ganglia were positioned on a cryostat chuck and frozen in place using OCT (Miles). Blocks were allowed to equilibrate at $-20^{\circ} \mathrm{C}$, then $8-\mu \mathrm{m}$ sections were cut on a Slee cryostat and mounted by drying onto gelatin-coated slides. Tissues were fixed to the slides by immersion in $10 \%$ buffered formalin, washed in PBS with the addition of $1 \mathrm{mg} /$ $\mathrm{ml}$ glycine, and incubated in a blocking solution consisting of $10 \%$ goat serum and $0.3 \%$ saponin in PBS. Primary antibody, diluted in blocking solution, was applied to the sections, and the slides were incubated in a humidified chamber overnight at $4^{\circ} \mathrm{C}$. The next day, sections were washed in PBS, rinsed in blocking solution, incubated in secondary antibody for $2-4 \mathrm{hr}$ at room temperature, washed briefly in PBS, incubated in $1 \mathrm{mg} / \mathrm{ml}$ diaminobenzidine (DAB; Sigma), reacted with the addition of $0.003 \% \mathrm{H}_{2} \mathrm{O}_{2}$, and coverslipped with $90 \%$ glycerol in PBS. Sections were viewed on a Leitz Diaplan microscope using Nomarski optics.

Grasshopper embryos, obtained from a colony of Schistocerca americana maintained at the University of Utah, were prepared for immunohistochemistry by dissecting them from their eggs into Ringer's solution. Embryos were staged by comparison with the staging series prepared by Bentley et al. (1979). Embryos were transferred into $2 \%$ paraformaldehyde in Millonig's buffer and fixed for $60 \mathrm{~min}$. Whole embryos were washed, blocked, and incubated in antibodies using the same protocol as was used for sections of adult ganglia, then were mounted in $90 \%$ glycerol and viewed using Nomarski optics or epifluorescence illumination.

Primary antibody dilutions were as follows: The 7F7 monoclonal antibody was used at a dilution of 1:500 in blocking solution, 5B12 hybridoma supernatant (obtained from Dr. Mark Meyer at the University of Washington) was used at a dilution of $1: 3$, and fluorescein isothiocyanate (FITC) conjugated anti-horseradish peroxidase (Jackson Immunoresearch) was used at 1:500. Secondary antibodies [rhodamine isothiocyanate- (RITC), or HRP-conjugated goat anti-mouse antibodies; Jackson Immunoresearch] were applied at a dilution of 1:200 in blocking solution. HRP-conjugated secondary antibodies were visualized by incubation $1 \mathrm{mg} / \mathrm{ml}$ DAB followed by reaction with $0.003 \%$ $\mathrm{H}_{2} \mathrm{O}_{2}$.

Electron microscopy. Thirty-five percent grasshopper embryos were dissected under sterile conditions into embryo culture medium containing 50\% Schneider's Drosophila medium (Gibco), 40\% minimum essential medium (MEM; Gibco), 10\% heat-treated fetal calf serum (Hyclone), $1.5 \mathrm{mg} / \mathrm{ml}$ bovine insulin (Sigma), $0.03 \mathrm{mg} / \mathrm{ml}$ glycine (Sigma), $0.001 \mathrm{mg} / \mathrm{ml}$ juvenile hormone (Sigma), $0.001 \mathrm{mg} / \mathrm{ml} \beta$-ecdyste- 
rone (Sigma), and 10,000 U/ml antimycotic/antibiotic (Sigma). Live embryos were pinned onto glass slides, then incubated in embryo culture medium in the presence of $7 F 7$ monoclonal antibodies $(1: 100)$ at $30^{\circ} \mathrm{C}$ for $2 \mathrm{hr}$. Embryos were rinsed in fresh medium and incubated in culture medium with the addition of secondary antibody (HRP-conjugated goat anti-mouse, diluted 1:50). Embryos were fixed in $2 \%$ paraformaldehyde and $0.5 \%$ glutaraldehyde for $30 \mathrm{~min}$, washed, intensified by incubation in $0.2 \%$ cobalt chloride, and reacted with $\mathrm{DAB}$ with the addition of $\beta$-D-glucose and glucose oxidase to provide the $\mathrm{H}_{2} \mathrm{O}_{2}$ necessary for the reaction (Watson and Burrows, 1981). Embryos were postfixed in $1 \%$ paraformaldehyde and $1 \%$ glutaraldehyde, en bloc stained with uranyl acetate, dehydrated in graded ethanol, and embedded in Spurr's resin. Sections were cut on a Reichardt-Jung microtome, mounted on Formvar-coated single slot grids, and vicwed in a Philips 201 electron microscope.

Immunoprecipitation. The antigen recognized by the 7F7 antibody was immunoprecipitated from a membrane preparation made from $40 \%$ grasshopper embryos. Forty percent grasshopper embryos were dissected into cold RPMI 1640 (Cellgro) with the addition of $6 \mathrm{mg} / \mathrm{ml}$ glycine. Embryos were washed in $10 \mathrm{~mm}$ triethanolamine (TEA) and 5 mM EDTA with the addition of protease inhibitors and homogenized. The homogenate was spun at $1000 \times g$ to remove the nuclei and cellular organelles, then the supernatant was removed and spun at $200,000 \times$ $g$ to pellet the membrane fraction. The pellet was resuspended in 10 mM TEA and radiolabeled with ${ }^{125}$ I using lactoperoxidase (Haustein et al., 1975). Immunoprecipitation was performed using previously described methods (Patel et al., 1987); the isolated antigen was run on a $12.5 \%$ polyacrylamide gel in the presence of sodium dodecyl sulfate (SDS) and visualized by overnight exposure to Kodak X-OMAT X-ray film using an intensifier screen.

Affinity purification. A monoclonal affinity column column was prepared by coupling 7F7 antibodies to protein G-coated Sepharose beads (Pharmacia) using dimethylpimilimidate (Harlow and Lane, 1988). The antigen was extracted from grasshopper embryo lysate made from $40 \%$ grasshopper embryos. Lysate was made by extruding the embryos from their eggs into PBS with added protease inhibitors (PI; see above). Crushed embryos were separated from yolk by centrifugation at 17,000 $\times g$ for $30 \mathrm{~min}$; the resulting pellet was resuspended in lysate buffer [10 mм TEA, 0.15 м NaCl, 2\% Nonidet P-40 (NP-40), 0.5\% deoxycholate (DOC), $\mathrm{pH} \mathrm{8.2,} \mathrm{plus} \mathrm{PI}]$ and stirred for $60 \mathrm{~min}$ at $4^{\circ} \mathrm{C}$. The suspension was centrifuged again to remove the insoluble fraction, and the resulting supernatant was filtered through gauze, then frozen at $70^{\circ} \mathrm{C}$ until necded. Thawed lysate was spun at $200,000 \times g$, then passed over the column at a rate of $200 \mathrm{ml} / 24 \mathrm{hr}$. After exposure to lysate, the column was washed 4 times at pH 8.2 using the following buffers: (1) $10 \mathrm{mM}$ TEA, $0.15 \mathrm{M}, \mathrm{NaCl}, 1 \% \mathrm{NP}-40$, plus PI; (2) $10 \mathrm{~mm}$ TEA, $0.15 \mathrm{~m} \mathrm{NaCl}$, plus PI; (3) $10 \mathrm{~mm}$ TEA, $0.15 \mathrm{M} \mathrm{NaCl}, 0.5 \% \mathrm{DOC}$, plus PI; and (4) $10 \mathrm{~mm}$ TEA, $1.0 \mathrm{M} \mathrm{NaCl}, 1 \% \mathrm{NP}-40$, plus PI. The 7F7 antigen was eluted from the column under high-pH conditions (elution buffer contained $50 \mathrm{mM}$ TEA, $0.15 \mathrm{~m} \mathrm{NaCl}, 1 \% \mathrm{NP}-40, \mathrm{pH} 11.5$, plus PI) in $1-\mathrm{ml}$ aliquots, precipitated using trichloroacetic acid, and run on $10 \%$ polyacrylamide gels in the presence of SDS. Proteins were visualized using Coomassie blue or silver staining. Molecular-weight standards (Bio-Rad, 14.5-200 $\mathrm{kDa}$ ) were run with each gel.

Generation of polyclonal serum. Polyclonal antibodies were produced to isolated column fractions by immunizing $\mathrm{Balb} / \mathrm{C}$ femalc micc with whole-column fractions (approximately $10 \mu \mathrm{g}$ protein per injection). Column fractions were precipitated with trichloroacetic acid, washed in acetone, then lyophilized. Dry proteins were resuspended in PBS, emulsified with an equal volume of Freund's adjuvant, then injected intraperitoneally. The first injection was made using Freund's complete adjuvant; subsequent injections 2 weeks apart were made using incomplete adjuvant. Serum was collected from mice 1 week after each injection by bleeding from the tail vein, then used at a dilution of 1:500 to label grasshopper embryos as above.

\section{Results}

Immunohistochemical characterization of the $7 F 7$ antigen Initial screening of hybridoma culture supernatant containing the 7F7 antibody suggested that the antibody recognized some specific elements of the embryonic grasshopper CNS. To determine what cells or types of cells the antibody was recognizing, we labeled sections through adult segmental ganglia and whole- mount embryos at $40 \%$ of development using the 7F7 antibody and compared the labeling pattern to that obtained using previously characterized antibodies.

In adult segmental ganglia, the 7F7 antibody labels a discrete ring surrounding the central neuropil (Fig. $1 A$ ). This ring corresponds to the position of the neuropilar glial cells, a ring of glial cell bodies surrounding the central neuropil that extend processes into the neuropil (Hoyle, 1986). Double labeling using Hoechst 33258, a fluorescent nuclear dye, and the 7F7 antibody indicates that 7F7 antibody labeling colocalizes with the position of a ring of glial nuclei surrounding the neuropil, but does not label other glial cell bodies, such as the perineural glial cells, which are located external to the neuronal cell bodies (data not shown). 7F7 antibody labeling of adult ganglia is not confined to the region where the neuropilar glial cell bodies are located, but extends into the central neuropil and along the longitudinal connectives and segmental nerves (Fig. $1 A, C$ ). Within the central neuropil, 7F7 antibody labeling was restricted to axonal tracts; synaptic neuropil was unlabeled. Labeling was continuously associated with axons as they coursed through the central neuropil and then entered the longitudinal connectives, or as they passed laterally into the segmental nerves. Similar sections of adult ganglia were labeled with the 5B12 monoclonal antibody, which recognizes glial cells in cricket ganglia (Meyer et al., 1988) and which cross-reacts with grasshoppers (Meyer and Edwards, 1989). Comparison of the 7F7 antibody labeling pattern with the 5B12 antibody labeling pattern (Fig. 1) shows that there is overlap between the 2 labeling patterns, suggesting that the 2 antibodies are labeling some of the same structures. The 5B12 antibody strongly labels the neuropilar glial cells, which also label with the 7F7 antibody. The 5B12 antibody also labels some structures within the central neuropil, but does not label the longitudinal connectives. 5B12 antibody labeling in the central neuropil is restricted to sensory nerve tracts (Meyer et al., 1987); 7F7 antibody labeling is more widespread, suggesting it labels both sensory and motor tracts. This labeling may be of glial cell processes associated with individual axonal tracts.

7F7 antibody labeling was also compared to 5B12 antibody labeling in grasshopper embryos. During embryonic development, the labeling pattern using the $5 \mathrm{~B} 12$ antibody is different from the 7F7 antibody labeling pattern. The 5B12 antibody labels several axonal tracts within the anterior and posterior commissures (Meyer et al., 1988). The 5B12 antibody does not label the crescent-shaped structures described above, nor does it label any laterally positioned cell clusters (data not shown). While it is likely that the $5 \mathrm{~B} 12$ and 7F7 monoclonal antibodies both label glial cells, it is unlikely that, in developing embryos, the 2 antibodies are labeling the same glial cells.

The embryonic pattern of 7F7 antibody labeling was compared to labeling using antibodies against HRP (anti-HRP), which recognizes all insect neurons (Jan and Jan, 1982). The anti-HRP antibodies were directly coupled to an FITC tag; 7F7 antibody labeling was visualized by using an RITC-conjugated goat antimouse secondary antibody. A single thoracic ganglion of a double-labeled 35\% grasshopper embryo is illustrated in Figure 2. Anti-HRP antibodies label all neuronal cell bodies and axons within the segmental ganglia and interganglionic connectives of the developing CNS (Fig. $2 A$ ). Neuronal cell bodies are clustered in the region surrounding the intersection of anterior and posterior commissures with the longitudinal connective in each hemisegment and are strung along the length of the longitudinal connectives. There are several cell clusters located adjacent to 




Figure 1. Labeling of adult segmental ganglia using 7F7 and 5B12 monoclonal antibodies. Horizontal cryostat sections through adult segmental ganglia were labeled using the 7F7 $(A, C)$ or 5B12 $(B, D)$ monoclonal antibodies. $A$ and $B$, Low magnification views of whole sections of segmental ganglia illustrate that both the 7F7 and the 5B12 antibodies label the neuropilar glial ring surrounding the central neuropil (arrowheads). Both antibodies also label axonal tracts within the neuropil (asterisks). Scale bars, $100 \mu \mathrm{m}$. $C$, Higher magnification of the posterolateral edge of a ganglion labeled with the 7F7 antibody. Labeling is seen in the ring of neuropilar glial cells (arrowheads) and extending into the longitudinal connective ( $l$ ). Neuronal cell bodies $(n)$ are not labeled. Scale bar, $20 \mu \mathrm{m}$. $D$, Higher magnification of the posterolateral edge of a ganglion labeled with the $5 \mathrm{~B} 12$ antibody. Labeling is seen in the ring of neuropilar glial cells (arrowheads), but not in the longitudinal connective ( $l$ ) or associated with neuronal cell bodies $(n)$. Scale bar, $20 \mu \mathrm{m}$.

the labeled neuronal cell bodies in each hemiganglion that do not label with the anti-HRP antibodies. One cluster is positioned laterally in the hemiganglion, immediately anterior to the emerging segmental nerve (Fig. $2 A$ ); a second cluster is at the anteromedial edge of the ganglion, just medial to the longitudinal connective (Fig. $2 A$ ). Examination of the same ganglion labeled with the 7F7 antibody shows that these clusters do label with the 7F7 antibody (Fig. 2B). Additional 7F7 antibody-labeled cell clusters that do not label with anti-HRP antibodies are seen in association with the longitudinal connectives, located midway between 2 adjacent ganglia (Fig. $2 A, B$ ). At least one of these longitudinal connective-associated clusters appears to extend a lamella that lines part of the lateral edge of the connective (Fig. 2B). At this stage of development, the most prominent 7F7 antibody labeling appears in 2 roughly crescent-shaped patches that are positioned at the posterolateral margins of the anterior commissure. The identity of these structures will be discussed below. The patterns of labeling using anti-HRP and 7F7 antibodies appear to be nonoverlapping; that is, cells labeled with the 7F7 antibody are not labeled with anti-HRP. This nonoverlapping pattern suggests that the 7F7 antibody is labeling a non-neuronal population of cells that are likely to be glial cells.

\section{Developmental expression of the $7 F 7$ antigen}

$7 \mathrm{~F} 7$ antigen expression in the CNS first appears at $25 \%$ of development. At this time in development, antigen expression is apparent within the ventral neuroblast sheet, where labeling is seen around some, but not all, neuroblasts (Fig. $3 A$ ). Labeling appears most prominent around a single medial neuroblast in each hemiganglion, neuroblast 4-1 (see Doe and Goodman, 1985, for terminology). Light labeling is occasionally seen around neuroblasts 5-1 and 6-1, as well (data not shown). The labeling appears diffusely distributed around the edges of the neuroblasts, 
suggesting that it is the sheath cells surrounding the neuroblasts that are recognized by the antibody, and not the neuroblasts themselves. Additional labeling is also seen outside the CNS, primarily at the interface between the ectoderm and mesoderm in the developing limb buds.

By $30 \%$ of development, a prominent patch of labeling appears in the region of the embryo where the anterior commissure will form. Labeling is of 2 roughly crescent-shaped structures located at what will be the caudolateral margin of the anterior commissure, where the commissure will eventually intersect with the longitudinal connective (Fig. $3 B$ ). Focusing through the crescents using Nomarski optics shows that the 7F7 antibody labeling is restricted to lamellar processes extending dorsally and laterally from more ventrally and medially located cell bodies (Fig. 4). These lamellar processes wrap around 2 cell bodies lying medial to the developing longitudinal connective and immediately posterior to the presumptive location of the anterior commissure. At this point in development, no axons have yet entered the anterior commissure, suggesting that the crescents may delimit the caudal margin of this pathway. Based on their location in association with the anterior commissure and on their expression of a non-neuronal antigen, we have named the cells that give rise to the crescents the anterior commissural glial (ACG) cells.

7F7 antibody labeling is more extensive at 35\% of development. Labeling of the ACG crescents associated with the anterior commissure is apparent, as is labeling around the edges of a cluster of 6-8 cells positioned immediately rostral to the anterior commissure (Fig. $3 \mathrm{C}$ ). This cell cluster is positioned in such a way that it might mark the rostral edge of the anterior commissure. By this point in development, several axons have entered the commissure and have crossed the midline to the contralateral side of the ganglion. Additional labeled cell clusters are located more laterally in each hemiganglion and in association with the longitudinal connectives (Figs. $2 B, 3 C$ ). It is difficult to distinguish individual cells within these cell clusters based on their 7F7 antibody labeling, though the edges of the clusters are clearly labeled. This suggests either that the 7F7 antigen is regionally expressed on the edges of cells, or that the antibody is labeling a cell process that wraps around these cell clusters. Some of the labeled clusters extend labeled lamellar processes that line the edges of developing axon tracts. The cell cluster located lateral to the longitudinal connectives extends a lamellar process that lines the lateral edge of the developing connective; a similar lamella is seen lining the dorsal edge of the emerging segmental nerve (Figs. $2 B, 3 C$ ). These lamellae, positioned in association with developing nerve tracts, may act as borders or limiting membranes to confine axons to specific regions within the developing ganglion.

7F7 antibody labeling appears restricted to a subset of glial cells within the developing ganglion. Labeling appears in association only with the anterior commissure; there is no homologous labeling associated with the posterior commissure. Additionally, several identified glial cells within the developing ganglion do not express the antigen recognized by the 7F7 antibody. These include the segment boundary cell associated with the intersegmental nerve (Bastiani and Goodman, 1986) and several large glial cells lying on the dorsal surface of the developing longitudinal connectives (cf. Bastiani and Goodman, 1986; Jacobs and Goodman, 1989). On those glial cells that do label with the 7F7 antibody, antigen expression appears to be localized to specific regions of the cells. Labeling is seen around the
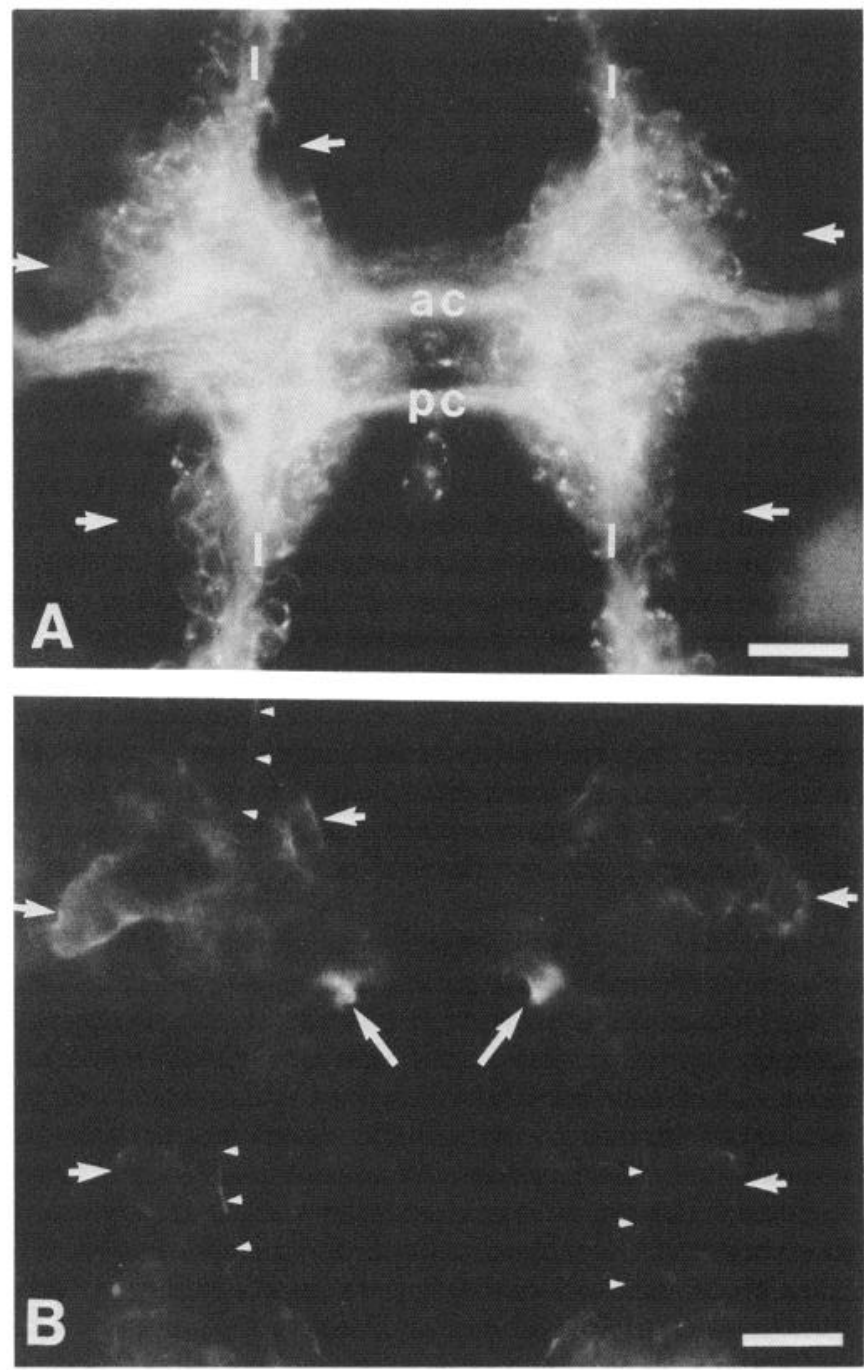

Figure 2. Single ganglion at $35 \%$ of embryonic development double labeled using anti-HRP and 7F7 antibodies. $A$. Anti-HRP labels all axons and neuronal cell bodies in the developing ganglion. Short arrows indicate the position of unlabeled cells that do label with the 7F7 antibody (see corresponding short arrows in $B$ ). ac, anterior commissure; $p c$, posterior commissure; $l$, longitudinal connectives. $B$, The $7 \mathrm{~F} 7$ antibody labels a population of cells that does not label with anti-HRP (short arrows). ACG crescents (long arrows) are present at the intersection of the anterior commissure and the longitudinal connectives. Small arrowheads indicate labeled lamellae extending along the longitudinal connectives. Scale bars, $30 \mu \mathrm{m}$. Anterior is toward the top of the figure; the midline of the animal runs through the center of each panel.

edges of cell clusters and on lamellar processes extending around adjacent cell bodies and along the edges of axonal tracts.

EM observation of a segmental ganglion at $40 \%$ of development labeled using the 7F7 antibody confirms that labeling is regionally localized on lamellar processes extending from lightly labeled or unlabeled cell bodies. Live embryos at $40 \%$ of development were labeled in culture using the 7F7 antibody, then were fixed and sectioned for EM observation. At this stage of development, labeling appears restricted to the margins of axonal tracts within the developing neuropil and extends along the edges of the longitudinal connectives and segmental nerves (Fig. 4A). The ACG crescents have shifted medially due to the broadening of the longitudinal connectives. EM observations 


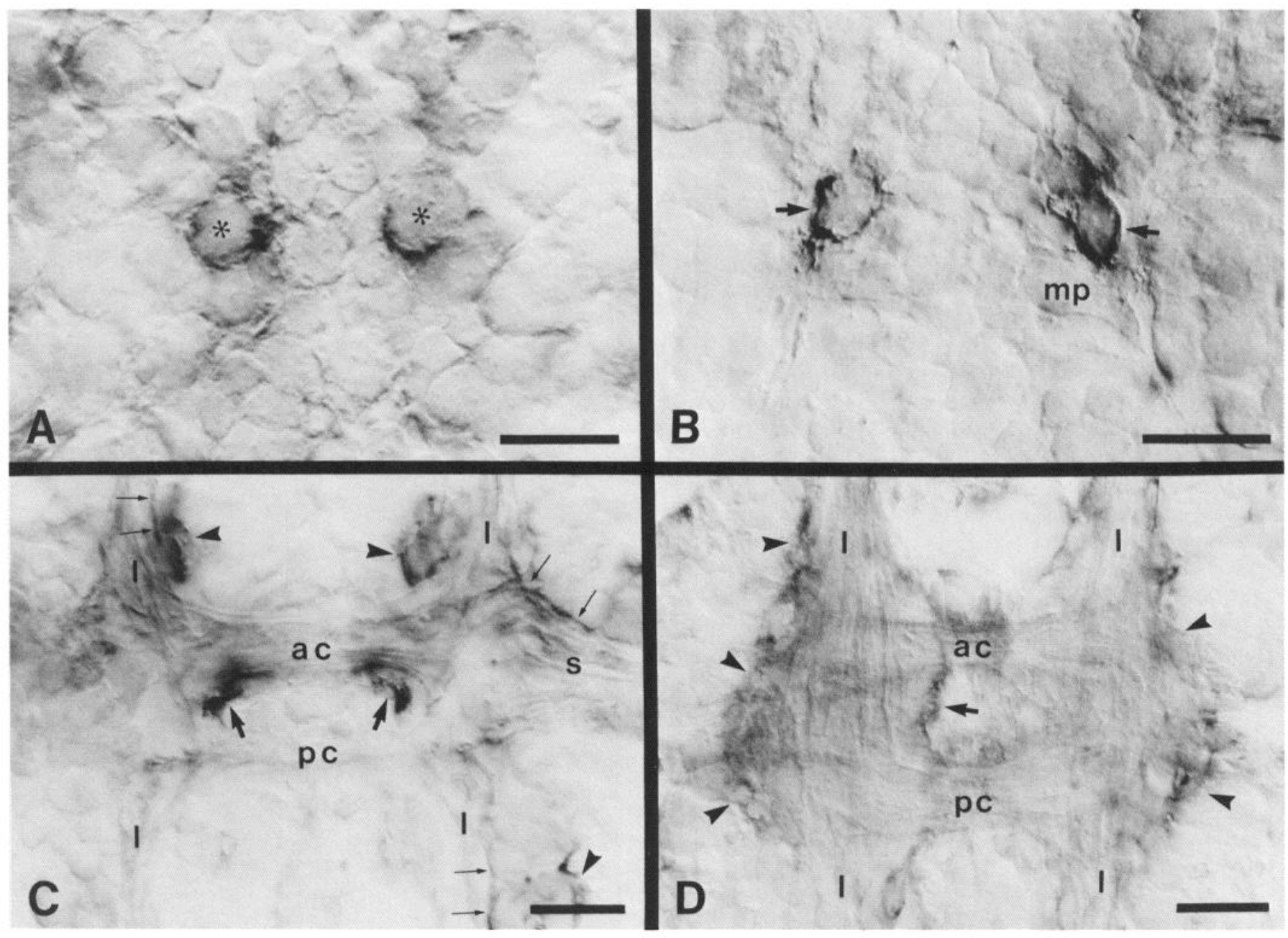

Figure 3. Developmental progression of 7F7 antibody labeling. A, A single thoracic segment in an embryo at $25 \%$ of development. The $7 \mathrm{~F} 7$ antibody labels around neuroblast 4-1 in the ventral neuroblast sheet in each hemiganglion (asterisks). B, A single segment at $30 \%$ of embryonic development. The 7F7 antibody labels the ACG crescents (arrows) on the dorsal surface of the CNS at what will be the caudolateral edge of the anterior commissure. At this point in development, the MP1 neuron $(\mathrm{mp})$ is just beginning to extend a growth cone. $C$, A single thoracic segment at 35\% of embryonic development. The 7F7 antibody labels the ACG crescents (large arrows) and clusters of cells located at the anterior margin of the anterior commissure and lateral to the longitudinal connective (arrowheads). Lamellae extend from some of these cell clusters to line the edges of the longitudinal connectives and the margin of the segmental $(s)$ nerve (paired small arrows). $D$, A single ganglion at $55 \%$ of development. The 7F7 antibody labels the lateral margin of the neuropil (arrowheads) and the medial edges of the longitudinal connective (arrow) as it passes through the ganglion. $a c$, anterior commissure; $p c$, posterior commissure; $l$, longitudinal connectives. Scale bars, $30 \mu \mathrm{m}$. In these 4 panels, anterior is at the top of the figure, and the midline of the animal runs down the center of each panel.

verify that the ACG cell bodies are located ventrally in the ganglion, adjacent to the midline (Fig. $4 \mathrm{C}$ ). The ACG cell bodies are electron lucent compared to surrounding cells, consistent with their being glial cells (Jacobs and Goodman, 1989). The surfaces of these cell bodies are lightly labeled with the 7F7 antibody, with labeling apparent only in a few patches on the surface of the cells (Fig. 4C). The ACG cell bodies extend labeled processes upward adjacent to the midline. These processes turn laterally and lie on the dorsal surface of several more dorsally positioned cells in sections adjacent to that illustrated in Figure $4 C$. The axons of the anterior commissure lie immediately anterior to this cell process and are separated from it by less than the diameter of a single axon. At this time in development, axons do not appear to lie in direct contact with the ACG cell process, though they are positioned so that the labeled process is within easy filopodial grasp. Laterally, a 7F7 antibody-labeled process appears to demarcate the lateral margin of the longi- tudinal connective (Fig. 4B). This process extends dorsally from the lateral edge of an unlabeled, electron-lucent glial cell body. Axons from the longitudinal connective run medial to this cell process, and filopodia extending from axons of the longitudinal connective appear to be in direct contact with the labeled process.

By $55 \%$ of development, 7F7 antibody labeling surrounds the developing neuropil, approximating the position of the neuropilar glial ring (Fig. 3D). There is also labeling lining the medial and lateral edges of the longitudinal connectives as they run between and through the ganglia. Labeling of the neuropil itself appears diffuse and not as dense as the more lateral labeling associated with the edges of the neuropil, and may correspond to labeling of glial processes extending centrally within the neuropil to surround and isolate individual axon tracts. This pattern of expression approximates that which will be seen in the adult segmental ganglia (cf. Fig. 1 $A, C$ ). 


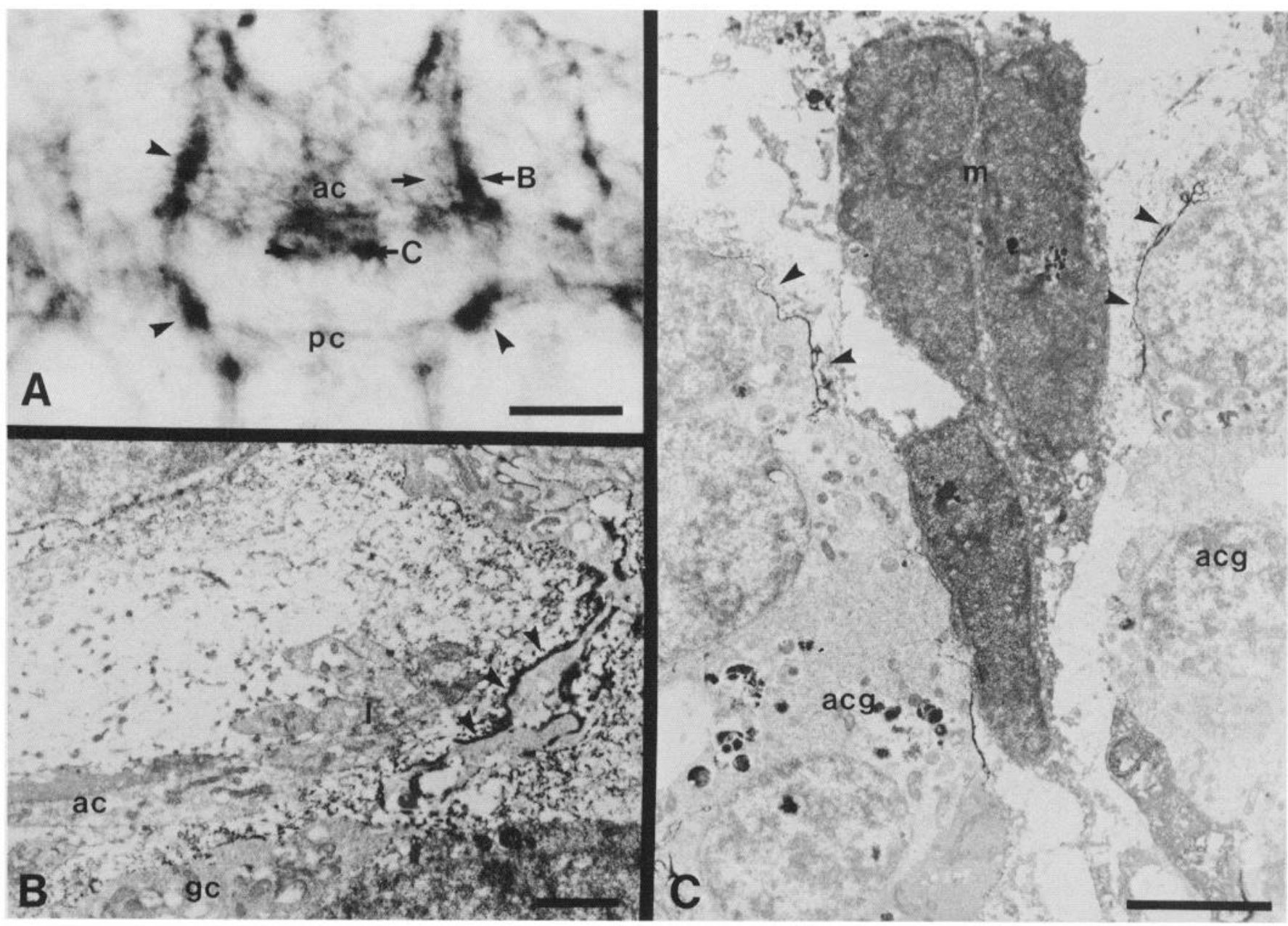

Figure 4. A, 7F7 antibody labeling of a single ganglion in a live embryo at $40 \%$ of development. The borders of the neuropil are outlined with 7F7 antibody labeling (arrowheads). Arrows at $B$ and $C$ demarcate the plane of section illustrated in the other panels. $a c$, anterior commissure; $p c$, posterior commissure. Scale bar, $30 \mu \mathrm{m}$. B. Electron micrograph of a laterally positioned, labeled lamella (arrowheads) extending dorsally from an unlabeled glia cell $(\mathrm{gc})$. The 7F7 antibody labels the surface of this lamella. Axons in the longitudinal connective $(l)$ run medially to this lamella. $a c$, anterior commissure. Scale bar, $2 \mu \mathrm{m}$. $C$, Electron micrograph of the anterior commissure glial cell bodies $(a c g)$ and their dorsally projecting lamellae (arrowheads). $m$, midline cells. Scale bar, $5 \mu \mathrm{m}$.

\section{Biochemical characterization of the $7 F 7$ antigen}

The antibody was used to immunoprecipitate the antigen from membranes obtained from grasshopper embryos at $40 \%$ of development. Membrane proteins were iodinated, then solubilized with detergent. The antigen was precipitated by binding to a preformed antibody complex composed of the 7F7 antibody and a rabbit anti-mouse secondary antibody. Following immunoprecipitation, the molecule recognized by the 7F7 antibody was analyzed by SDS-PAGE. Comparison of the results obtained using the 7F7 antibody to the results obtained from several other antibodies available in the laboratory shows that the 7F7 antibody recognized a single unique band having a molecular weight of $60 \mathrm{kDa}$ (Fig. 5). The antigen was purified using a monoclonal affinity column. The 7F7 antibody was coupled to Protein G-coated Sepharose beads, then lysate made from $40 \%$ grasshopper embryos was passed over the column. The antigen was eluted from the column at high $\mathrm{pH}$; eluted fractions were analyzed by SDS-PAGE. This single-step purification resulted in the isolation of a single prominent band having a molecular weight of $60 \mathrm{kDa}$ (Fig. 6). Based on the immunohistochemical localization of this antigen to specific regions of glial cells, the molecule recognized by the 7F7 antibody was named REGA-1, for regionally expressed glial antigen-1.

To confirm the identity of the purified molecule, polyclonal antibodies were generated in mice against whole-column fractions. Polyclonal serum was used to label whole-mount $35 \%$ grasshopper embryos and was compared to the labeling of agematched control embryos using the 7F7 monoclonal antibody. The polyclonal antibody labels $35 \%$ embryos in a pattern that is very similar to that obtained using the monoclonal antibody (Fig. 7). The polyclonal antibody labels the ACG crescents associated with the anterior commissure as well as a subset of cells within the developing ganglion. It also labels lamellar processes lining the lateral margins of the developing neuropil and longitudinal connectives. In general, labeling using the polyclonal antibody appears to be slightly more widespread than labeling using the monoclonal antibody, but the patterns of labeling using the 2 antibodies are very similar, suggesting that both antibodies are recognizing the same molecule. The more widespread labeling using the polyclonal may be due to a variety 


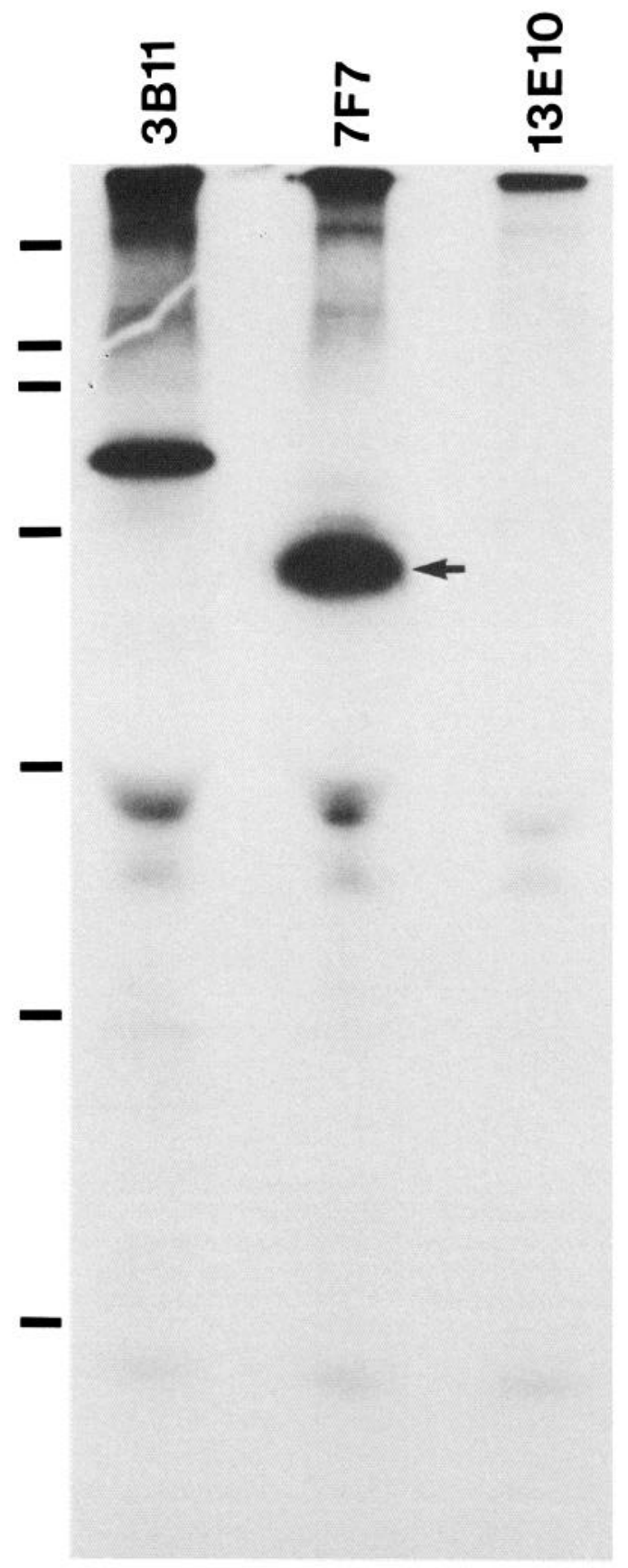

Figure 5. Autoradiograph of immunoprecipitation of REGA-1 from a membrane preparation made from $40 \%$ grasshopper embryos. The $7 F 7$ antibody recognizes a single unique band at $60 \mathrm{kDa}$ (arrow). $3 B 11$ is positive control (fasciclin l); $13 E 10$ is the negative control demonstrating nonspecific trapping by the preformed complex. Tick marks along the left are molecular-weight markers in descending order: 200, $116,97,66,43,31$, and $21.5 \mathrm{kDa}$.

of shared epitopes recognized by the polyclonal serum. The presence of the characteristic features of 7F7 monoclonal antibody labeling including the ACG crescents and lamellae associated with axonal tracts is seen following labeling using the polyclonal antibody. This suggests that the polyclonal antibody does recognize the REGA-1 molecule and confirms that the 60$\mathrm{kDa}$ band isolated by affinity purification is indeed REGA-1.

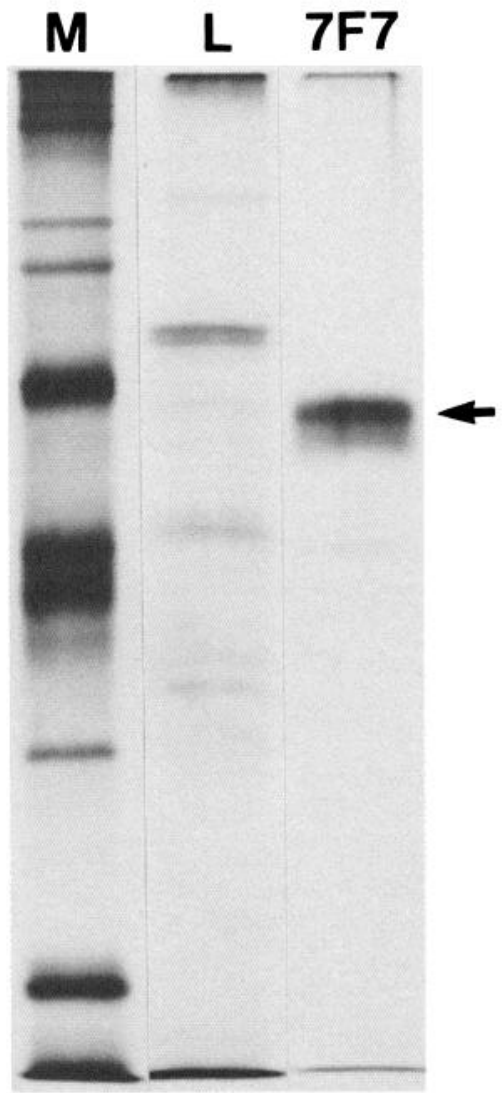

Figure 6. Silver-stained $10 \%$ polyacrylamide gel demonstrating affinity purification of REGA-1. REGA-1, the predominant band recognized by the $7 F 7$ antibody, has a molecular weight of $60 \mathrm{kDa}$ (arrow). $M$, molecular-weight markers; $L$, whole-embryo lysate. Molecular-weight marker weights are given in Figure 5, caption.

\section{Discussion}

REGA-1 is expressed on a subset of glial cells

REGA-1 is expressed on localized regions of a unique subset of non-neuronal cells in the developing nervous system of the grasshopper embryo. The 7F7 antibody, which recognizes REGA-1, labels known glial structures in adult segmental ganglia. This is shown both on the basis of localization of the labeling to a ring of cell bodies in the position of the neuropilar glial cells (Hoyle, 1986) and on the basis of labeling in regions recognized by the 5B12 antibody, a known insect glial label (Meyer et al., 1988). REGA-1 is present on clusters of cell bodies in the embryonic nervous system that are not recognized by the anti-HRP antibody, also suggesting its non-neuronal or glial expression. The morphology of some of the cells expressing REGA-1 is typical of glial morphology. Some cells extend processes that wrap around other cell bodies, as seen in the crescents; other cells or clusters of cells extend lamellae that line the edges of axonal pathways such as the longitudinal connective. These observations all suggest that REGA-1 is expressed on a population of glial cells within the developing CNS of grasshopper embryos.

REGA-1 expression is restricted to selected cell clusters within the developing CNS, which include some, but not all, of the CNS glial cells. Other known glial cells do not express REGA1 , including the well-described segment boundary cell (Bastiani and Goodman, 1986). The 7F7 antibody detects a molecular 
heterogeneity that was only presumed to exist within the glial population of the embryonic grasshopper nervous system. Morphological heterogeneity among glial cells has been extensively described in the adult insect CNS (Wigglesworth, 1959; Lane, 1974; Hoyle, 1986); there is no reason to suppose that a similar heterogeneity could not exist among embryonic glial cells. We suggest that the 7F7 antibody is able to detect such heterogeneity. It could be argued that the 7F7 antibody is recognizing differentiated glial cells arising from an undifferentiated glial precursor population. Yet, if this were the case, one would expect to see an increase in 7F7 antibody labeling throughout development as more and more mature glial cells are recruited from a precursor population. While this is to some extent true, as is seen in the increase of antigen expression in the neuropilar glial ring, certain identified glial cells, such as the segment boundary cell, do not express REGA-1. Additionally, there are entire populations of glial cells in the adult ganglion, such as the perineural glia, that also do not express REGA-1.

Glial heterogeneity may arise in several different ways. Distinct types of glial cells may arise from separate precursor populations, or they may arise from the same precursor and differentiate into different classes of glial cells as they mature and assume a glial phenotype. Previous work has described glial precursor cells located laterally within the neuroblast sheet (Doe and Goodman, 1985). 7F7 antibody labeling is apparent at early stages in the neuroblast sheet, but in a different position from that described for these other glial precursors. This suggests that there may be several distinct glial precursors, each of which gives rise to a different type of mature glial cell. This idea is further supported by progenitor labeling studies that can separate different populations of glial cells depending on their time of appearance (Shepherd and Bate, 1990).

This heterogeneity suggests that all glial cells are not created equal, and that they have features that may be differentiated both morphologically and immunologically. This, in turn, sets the stage for the potential use of glial cells as pathway markers for axon guidance. The glial cells expressing REGA-1 are ideal candidates to provide guidance cues for growing axons. The features that make them attractive for this purpose are their limited distribution, their appearance before the onset of axon outgrowth, and their intimate association with selected axonal pathways. The fact that they are not uniformly distributed along all pathways of axonal growth argues against the idea that they are simply providing is a favorable substrate for growth. Instead, our data support the idea that 7F7 antibody-labeled glial cells may play a more defined role in shaping the axonal pathways of the developing CNS.

\section{Glial cells expressing REGA-1 delineate the margins of some axonal pathways}

Glial cells expressing REGA-1 are positioned appropriately both spatially and temporally to play a role in establishing the location of some axonal pathways within the developing grasshopper CNS. REGA-1 expression appears early in the CNS, first around neuroblasts within the neuroepithelial sheet, then on 2 distinctive crescents arising from the ACG cells at the caudal border of the developing anterior commissure. The ACG crescents are located in a position to demarcate the caudal border of the anterior commissure; additionally, their appearance precedes the growth of axons into the anterior commissure. Cell processes expressing REGA-1 are also seen along the edges of the longitudinal connectives and the segmental nerves, sug-

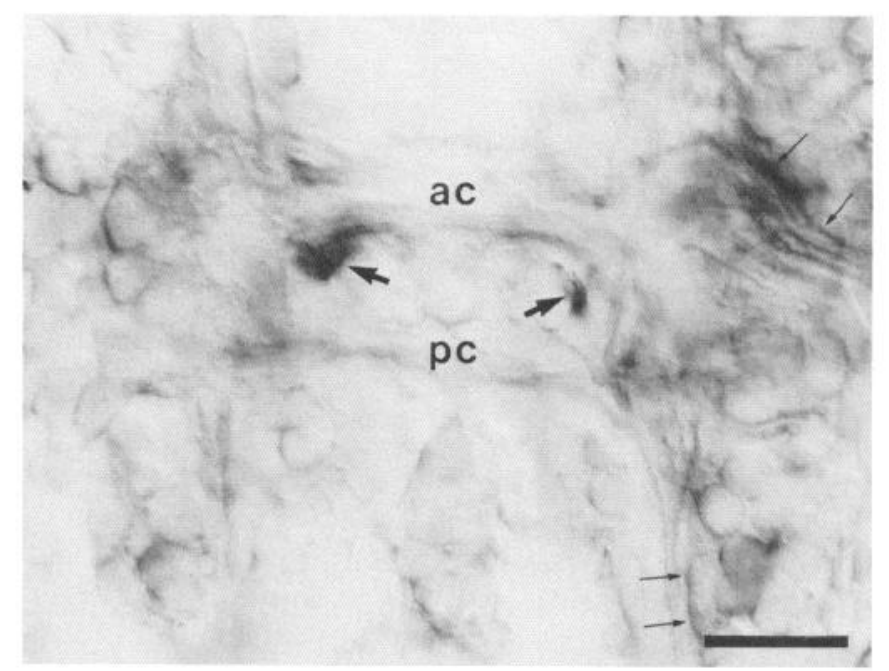

Figure 7. Immunohistochemical labeling of single ganglion at $35 \%$ of development using polyclonal antibody generated against column fractions isolated from 7F7 monoclonal antibody affinity column. Several features of the 7F7 antibody labeling pattern, such as the ACG crescents (arrows) and the lamellae lining the lateral edges of the longitudinal connectives and the segmental nerve (paired small arrows), are labeled using this polyclonal antibody. $a c$, anterior commissure; $p c$, posterior commissure. Scale bar, $30 \mu \mathrm{m}$.

gesting that labeled processes may play a role in marking the borders of these pathways, as well. Late in development and in adults, REGA-1 expression is limited to those glial cells that are associated with the central neuropil and with the longitudinal connectives and segmental nerves. In all of these regions, REGA-1 may be expressed only on those glial cells associated with axons.

The morphology of cells and cell clusters that express REGA-1 and are associated with axonal pathways suggests that these cells may play a role in defining or establishing the positions of selected axonal pathways within the developing CNS. In several cases, these cells have been seen to extend lamellae that line the edges of axonal pathways such as the anterior commissure and the longitudinal connectives. We suggest that these lamellae may act as borders or guard rails to delimit the margins of some axonal pathways, acting to prevent axons from growing outside of the appropriate regions. Similar structures have been identified in vertebrate embryos (Silver et al., 1987), and similar inhibitory functions have been ascribed to vertebrate CNS glial cells (Caroni and Schwab, 1988). As development proceeds, cells expressing REGA-1 develop into at least part of the neuropilar glial ring. In adults, this ring isolates a central core of nerve fibers from the surrounding neuronal cell bodies. Within the neuropil, REGA-1 expression appears associated only with axonal tracts and not with regions of synaptic neuropil, again potentially isolating axons from surrounding inappropriate areas. REGA-1 expression also extends out of the neuropil, but only in association with those axons that enter the longitudinal connectives or the peripheral nerves. All of these observations support the idea that cells expressing REGA-1 are likely to be those glial cells associated with axons. In addition, the interactions between axons and glial cells expressing REGA-1 may result in the segregation of axons away from inappropriate regions. 


\section{REGA-1 is regionally expressed on glial cells}

Our initial observations using light microscopy suggested that 7F7 antibody labeling was present only on lamellar processes extending from unlabeled cell bodies. Our EM observations have supported this initial observation, showing that REGA-1 appears regionally localized on glial cell processes closely associated with axon pathways, while the cell bodies giving rise to these processes either do not express this molecule or express it only in localized areas. These 2 observations suggest that REGA-1 is regionally expressed on only certain parts of glial cells, in particular, on the lamellar processes in close association with developing axonal pathways. Regional expression of cellsurface molecules has been described on developing Schwann cells (Martini and Schachner, 1986) and on developing neurons (Bastiani et al., 1987; Dodd et al., 1988). Regional expression of REGA-1 may reflect its role in the differentiation of specialized glial lamellae that may serve a specific function such as providing a border for axonal pathways.

Several other cell-surface molecules have been identified that are expressed on the surfaces of invertebrate glial cells. Our evidence suggests that REGA-1 is different from any previously described molecule. Its molecular weight $(60 \mathrm{kDa})$ sets it apart from the antigen recognized by the 5B12 antibody, which has a molecular weight of $185 \mathrm{kDa}$ (Meyer et al., 1988) and which has a different expression pattern than that demonstrated by REGA-1. Its molecular weight and its expression pattern are also different from neuroglian, a molecule with variable weights of 180,167 , and $155 \mathrm{kDa}$, which is expressed on neurons and glia in Drosophila (Bieber et al., 1989). Given that its expression appears limited to glial cells, REGA-1 is unlikely to be a homophilic adhesion molecule like neuroglian. It may, however, play a role in heterophilic interactions between neurons and glia, possibly signaling the location of axonal pathways to the appropriate neurons. Another possibility is that expression of REGA-1 results in establishing a nonpermissive substrate for axon growth (Caroni and Schwab, 1988), thus limiting axons to grow within certain regions. If, early in development, glial cell processes expressing REGA-1 act as borders to delimit the edges of developing axonal pathways such as the anterior commissure and the longitudinal connective, then the REGA-1 molecule may have an inhibitory function in that it may act to discourage axons from crossing such a border.

\section{References}

Bastiani MJ, Goodman CS (1986) Guidance of neuronal growth cones in the grasshopper embryo. III. Recognition of specific glial pathways. J Neurosci 6:3542-3551.

Bastiani MJ, Harrelson AL, Snow PM, Goodman CS (1987) Expression of fasciclin I and II glycoproteins on subsets of axon pathways during neuronal development in the grasshopper. Cell 48:745-755.

Bate CM (1976) Embryogenesis of an insect nervous system I: a map of the thoracic and abdominal neuroblasts in Locusta migratoria. $\mathbf{J}$ Embryol Exp Morphol 35:107-123.

Bentley DH, Keshishian H, Shankland M, Toroian-Raymond A (1979) Quantitative staging of embryonic development of the grasshopper, Schistocerca nitens. J Embryol Exp Morphol 54:47-74.

Bieber AJ, Snow PM, Hortsch M, Patel NH, Jacobs JR, Traquina ZR, Schilling J, Goodman CS (1989) Drosophila neuroglian: a member of the immunoglobulin superfamily with extensive homology to the vertebrate neural adhesion molecule L1. Cell 59:447-460.

Campos-Ortega JA, Hartenstein V (1985) The embryonic development of Drosophila melanogaster. Berlin: Springer.

Caroni P, Schwab ME (1988) Two membrane protein fractions from rat central myelin with inhibitory properties for neurite growth and fibroblast spreading. J Cell Biol 106:1281-1288.

Dodd J, Morton SB, Karagogeos D, Yamamoto M, Jessell TM (1988)
Spatial regulation of axonal glycoprotein expression on subsets of embryonic spinal neurons. Neuron 1:105-116.

Doe CQ, Goodman CS (1985) Early events in insect neurogenesis. I. Developmental and segmental differences in the pattern of neuronal precursor cells. Dev Biol 111:193-205.

Doe CQ, Kuwada JY, Goodman CS (1985) From epithelium to neuroblasts to neurons: the role of cell interactions and cell lineage during insect neurogenesis. Philos Trans R Soc Lond [Biol] 312:67-81.

Goodman CS, Bastiani MJ, Doe CQ, du Lac S, Helfand SL, Kuwada JY, Thomas JB (1984) Cell recognition during neuronal development. Science 225:1271-1279.

Harlow E, Lane D (1988) Antibodies: a laboratory manual. Cold Spring Harbor, NY: Cold Spring Harbor Laboratory.

Haustein K, Marchalonis JJ, Harris AW (1975) Immunoglobulin of T lymphoma cells. Biosynthesis, surface representation and partial characterization. Biochemistry 14:1826-1834.

Hoyle G (1986) Glial cells of an insect ganglion. J Comp Neurol 246: 85-103.

Jacobs JR, Goodman CS (1989) Embryonic development of axon pathways in the Drosophila CNS. I. A glial scaffold appears before the first growth cones. J Neurosci 9:2402-2411.

Jan LY, Jan YN (1982) Antibodies to horseradish peroxidase as specific neuronal markers in Drosophila and in grasshopper embryos. Proc Natl Acad Sci USA 79:2700-2704.

Johnson EM Jr, Taniuchi M, DiStefano PS (1988) Expression and possible function of nerve growth factor receptors on Schwann cells. Trends Neurosci 11:299-304.

Kohler G, Milstein C (1975) Continuous cultures of fused cells secreting antibody of predefined specificity. Nature 256:495-497.

Lane NJ (1974) The organization of insect nervous systems. In: Insect neurobiology (Treherne JE, ed), pp 1-71. Oxford: North-Holland.

Martini R, Schachner M (1986) Immunoelectron microscopic localization of neural cell adhesion molecules (L1, N-CAM, and MAG) and their shared carbohydrate epitope and myelin basic protein in developing sciatic nerve. J Cell Biol 103:2439-2448.

Meyer MR, Edwards JS (1989) Neuroblasts express glia-associated antigens during insect embryonic development. Soc Neurosci Abstr 15:598.

Meyer MR, Reddy GR, Edwards JS (1987) Immunological probes reveal spatial and developmental diversity in insect neuroglia. J Neurosci 7:512-521.

Meyer MR, Brunner P, Edwards JS (1988) Developmental modulation of a glial cell-associated glycoprotein, 5B12, in an insect, Acheta domesticus. Dev Biol 130:374-391.

Patel NH, Snow PM, Goodman CS (1987) Characterization and cloning of fasciclin III: a glycoprotein expressed on a subset of neurons and axon pathways in Drosophila. Cell 48:975-988.

Poston MR, Fredieu J, Carney PR, Silver J (1988) Roles of glia and neural crest cells in creating axon pathways and boundaries in the vertebrate central and peripheral nervous systems. In: The making of the nervous system (Parnavelas JG, Stern CD, Stirling RV, eds), pp 282-313. Oxford: Oxford UP.

Radojcic T, Pentreath VW (1979) Invertebrate glia. Prog Neurobiol 12:115-179.

Shepherd D, Bate CM (1990) Spatial and temporal patterns of neurogenesis in the embryo of the locust (Schistocerca gregaria). Development 108:83-96.

Silver J, Rutishauser U (1984) Guidance of optic axons in vivo by a preformed adhesive pathway on neuroepithelial endfeet. Dev Biol 106:485-499.

Silver J, Lorenz SE, Wahlsten D, Coughlin J (1982) Axonal guidance during development of the great cerebral commissures: descriptive and experimental studies, in vivo, on the role of preformed glial pathways. J Comp Neurol 210:10-29.

Silver J, Poston M, Rutishauser U (1987) Axon pathway boundaries in the developing brain. I. Cellular and molecular determinants that scparate the optic and olfactory projections. J Neurosci 7:2264-2272.

Watson AHD, Burrows M (1981) Input and output synapses on identified motor neurons of a locust revealed by the intracellular injection of horseradish peroxidase. Cell Tissue Res 215:325-332.

Wetts R, Serbedzija GN, Fraser SE (1989) Cell lineage analysis reveals multipotent precursors in the ciliary margin of the frog retina. Dev Biol 136:254-263.

Wigglesworth VB (1959) The histology of the nervous system of an insect, Rhodnius prolixus (Hemiptera). II. The central ganglia. Q J Microsc Sci 100:299-313. 